\title{
AN ELASTIC-VISCO-PLASTIC DEFORMATION MODEL OF AL-LI WITH APPLICATION TO FORGING
}

\author{
L. B. BORKOWSKI, J. A. SHARON, \& A. STAROSELSKY \\ United Technologies Research Center, East Hartford, CT.
}

\begin{abstract}
Recent alloy developments have produced a new generation of $\mathrm{Al}-\mathrm{Li}$ alloys that provide not only weight savings, but also many property benefits such as excellent corrosion resistance, good spectrum fatigue crack growth performance, a good strength and toughness combination and compatibility with standard manufacturing techniques. The forging of such alloys would lead to mechanical properties that closely match the aircraft engine requirements including lower weight, improved performance and a longer life. As a result, detailed analyses need to be performed to determine which material properties are best suited for a specific structure and how to achieve the required mechanical and damage tolerant properties during material processing.

We developed an integrated physics-based model for prediction of microstructure evolution and material property prediction of third-generation Al-Li alloys. In order to develop such a model, an elastic-plastic crystal plasticity model is developed and incorporated in finite element software (ANSYS). The model accounts for microstructural evolution during non-isothermal, non-homogeneous deformation and is coupled with the damage kinetics. Our model bridges the gap between dislocation dynamics and continuum mechanics scales.

Model parameters have been calibrated against lab tests including micropillar in-situ simple compression tests of Al-Li alloy 2070. Numerical predictions are verified against the lab results including stress-strain curves and crystallographic texture evolution.

Keywords: crystallographic texture, light weight alloys, material characterization, material processing, micro-scale testing
\end{abstract}

\section{INTRODUCTION}

Aircraft weight is an important factor in fuel economy. Even a modest decrease in airplane weight reduces the fuel consumption and hence, allows increase in the maximum flight range, which in turn makes longer direct flights possible, avoiding extra take offs and landings, increasing engine reliability, reducing maintenance cost, not mentioning the additional fuel savings. As a rule-of-thumb, a $1 \%$ weight reduction in the gross weight of an empty aircraft corresponds to $0.25 \%-0.75 \%$ reduction in fuel consumption $[1,2]$. Our overall goal is to achieve weight reductions possible through material swaps in aircraft engines.

The first component in a turbofan engine is a fan composed of fast rotating blades. Currently most of the blades of the fan are made of titanium. There is a drive to develop them from lighter materials, such as aluminum alloys. The current design of large fan blades in commercial turbines is generally too heavy and generates excessively high stresses on the component and the surrounding structures. Weight reduction afforded by a switch to Al-Li alloys would resolve both of these issues.

The fan blades are the subject of significant temperature variation, high inertia stresses and a centripetal force through its root. Furthermore, these components must last for at least 10,000 flights, displaying sufficient flexibility and damage tolerance to non-interruptive operation under severe environmental, thermal, and the occasional dynamic (bird impact) conditions. The development and characterization of forged $\mathrm{Al}-\mathrm{Li}$ alloys are needed to enable the significant increase of engine reliability and reduced fuel consumption that would come with their deployment in engines. Given the anisotropy in properties and the relative 
immaturity of these alloys for turbine engine applications, much work still needs to be done to build the capability to predict forged Al-Li structural properties as a result of the material processing parameters.

Experience from the 1990's showed significant issues with Al-Li due to high planar anisotropy, unusual crack paths, and lack of thermal stability. However, recently, there has been a renewed interest in the new generation of $\mathrm{Al}-\mathrm{Li}$ alloys that eliminated or lessened these manufacturing concerns. This new interest is being driven by the challenge to meet significantly higher performance requirements demanded by the next generation of developed commercial aircraft engines [1-3]. High damage tolerance is particularly important for airframe structures and hence of primary interest to airframe manufacturers.

Predictive methodologies for Al-Li need to fully couple material processing parameters (forging and heat treatment), forged part mechanical properties, and damage tolerance required by turbine engine service conditions. The developed and implemented microstructure-based crystal plasticity computational framework allows predicting the effect of local morphology on the mechanical behavior of the components. Such a model requires accurate measurements of single crystal elastic-plastic properties as well as dislocation density distributions and its incorporation in the part level polycrystalline model. Values of these model parameters are estimated from calibration experiments (compression, tensile) for both single crystal and polycrystalline specimens under a wide range of thermal mechanical conditions. The constitutive equations are implemented in finite element software as a user routine $[4,5]$ and used for the modelling of the microstructure and properties evolution during the forging procedure and for the final part design optimization. The predictive accuracy of the model in these domains is being quantified through validation testing.

\section{CONSTITUTIVE MODEL}

The overall polycrystalline plastic response is taken as a sum of responses of each of the many single crystals that comprise the representative volume element (RVE). Material behavior is modeled by FEM where each element quadrature point represents an RVE and where both compatibility and equilibrium are satisfied. The deformation of a crystal is taken as the combined contributions from an overall elastic distortion of the lattice and plastic deformation.

The governing variables in the constitutive model are taken as: (1) Cauchy stress T, (2) total deformation gradient, $\mathbf{F}$ and (3) plastic deformation gradient $\mathbf{F}^{\mathbf{p}}$ with $\operatorname{det}_{\mathbf{F}}^{\mathbf{p}}=1$. Each crystal slip system is specified by a unit normal $\mathbf{n}_{0}^{\alpha}$ to the slip plane, and a unit vector $\mathbf{m}_{\mathbf{0}}^{\alpha}$ denoting the slip direction. We define twelve octahedral $\{111\}<110>$ slip systems to be operative. The elastic deformation gradient is defined by decomposition of the total deformation gradient as follows: $\mathbf{F}=\mathbf{F}^{\mathrm{e}} \mathbf{F}^{\mathrm{p}}$ where $\operatorname{det} \mathbf{F}^{\mathrm{e}}>0 . \mathbf{F}^{\mathrm{e}}$ describes the elastic distortion of the lattice and gives rise to the stress $\mathbf{T}$. For metallic materials the constitutive equation for the second Piola-Kirchhoff stress tensor is taken as a linear relation

$$
\mathbf{T}^{*}=\mathbf{C}\left[\mathbf{E} *-\mathbf{A} \cdot\left(\Theta-\Theta_{0}\right)\right] ; \quad \mathbf{E}^{*}=\frac{1}{2}\left[\mathbf{F}^{\mathrm{eT}} \mathbf{F}^{\mathrm{e}}-\mathbf{1}\right]
$$

where $\mathbf{A}$ is the second order thermal expansion tensor, $\mathbf{C}$ is the temperature-dependent anisotropic elasticity tensor of the fourth rank, $\Theta$ is the current temperature and $\Theta_{0}$ is the reference temperature. Deformation gradients, stress and strain tensors are second order tensors and represented by (3 by 3 ) matrices.

The Cauchy stress tensor is calculated as follows:

$$
\mathbf{T}=\frac{1}{\operatorname{det}\left(\mathbf{F}^{\mathrm{e}}\right)} \mathbf{F}^{\mathbf{e}} \mathbf{T}^{*} \mathbf{F}^{\mathrm{e} \mathbf{T}}
$$


The evolution equation for the viscoplastic deformation gradient is given by the flow rule:

$$
\dot{\mathbf{F}}^{\mathbf{p}}=\mathbf{L}^{\mathbf{p}} \mathbf{F}^{\mathbf{p}} ; \quad \text { where } \quad \mathbf{L}^{\mathbf{p}}=\sum_{\text {slip systems }} \dot{\gamma}^{a} \mathbf{S}^{a} \quad \text { and } \quad \mathbf{S}^{\alpha}=\mathbf{m}^{a} \otimes \mathbf{n}^{\alpha}
$$

The shear rate along each slip system, $\dot{\gamma}^{\alpha}$, is given in terms of the resolved shear stress (RSS), which is the projection of tensor $\mathbf{T}^{*}$ on a slip system $\alpha\left(\tau^{\alpha}=\mathbf{T}^{*}: \mathbf{S}^{\alpha}=\mathbf{T}^{*}:\left(\mathbf{m}_{0}^{a} \otimes \mathbf{n}_{0}^{\alpha}\right)=\left(\mathbf{T}^{*} \mathbf{n}_{0}^{a}\right) \cdot \mathbf{m}_{0}^{a}\right)$, slip system resistance and equilibrium back stress. Evolution of crystallographic texture is explicitly defined by the elastic part of the deformation gradient.

$$
\mathbf{m}_{\mathbf{t}}^{\alpha}=\mathbf{F}^{\mathbf{e}} \mathbf{m}_{\mathbf{0}}^{\alpha} ; \quad \mathbf{n}_{\mathbf{t}}^{\alpha}=\mathbf{F}^{\mathbf{e}-\mathbf{T}} \mathbf{n}_{\mathbf{0}}^{\alpha}
$$

Particular expression for shear rates is [4]:

$$
\left\{\dot{\gamma}^{c}\right\}^{\alpha}=\dot{\gamma}_{0}\left(\frac{\rho_{m}^{\alpha}}{\rho_{0}}\right)\left|\frac{\tau^{\alpha}-\omega^{\alpha}}{s^{\alpha}}\right|^{n} \operatorname{sgn}\left(\tau^{\alpha}-\omega^{\alpha}\right) \exp \left(-\frac{Q}{k \Theta}\right)
$$

where $\tau^{\alpha}$ is the RSS, $\omega^{\alpha}$ is a back stress and $S^{\alpha}$ is the deformation resistance of $\alpha$-th slip system. Arrhenius term allows accounting for the temperature changes. Next, latent hardening evolution has been described by modifying Asaro rule $\dot{s}^{\alpha}=h_{0}\left(1-\frac{s^{\alpha}}{s^{*}}\right)^{p} \sum_{\beta} h^{\alpha \beta}\left|\dot{\gamma}^{\beta}\right|$, with hardening matrix $h^{\alpha \beta}=\left\{q+(1-q) \delta^{\alpha \beta}\right\}$ for temperature dependent $h_{0}$ and $s^{*}$. The back stress has a limiting saturation value, $\omega_{\infty}=\frac{c_{1}}{c_{2}}$, corresponding to the end of the primary creep stage, which evolves according to the following relationship [4-6]: $\dot{\omega}^{\alpha}=c_{1} \dot{\gamma}^{\alpha}-c_{2}\left|\dot{\gamma}^{\alpha}\right| \omega^{\alpha}$. The back stress requires two additional coefficients, $c_{1}$ and $c_{2}$, that are explicit functions of temperature. It is important to note that hardening terms indirectly account for rafting process and microstructure evolution during the first stage of viscoplastic deformation. Results reported in this paper have been obtained by using the hardening expressions shown above. We postulate that dislocation generation rate is proportional to the entropy production. Using concepts from chemical kinetics we have chosen to represent the evolution as two body interactions. We assume that dislocation immobilization takes place when two corresponding dislocation loops interact with each other [4, 7]. For the sake of simplicity, prediction of the texture evolution during rolling operation was obtained with $\rho_{m}^{\alpha} / \rho_{0}=1$, which affects rate of relaxation but does not affect texture evolution.

\section{ELASTIC MODULI OF AA2070 FROM SINGLE CRYSTAL MICROPILLAR COMPRESSION}

\subsection{Experimental}

Single crystal data for AA2070 is sparse so micromechanical testing was employed to extract single crystal properties from small scale test structures fabricated within the individual grains of a polycrystalline billet. Specifically, micron size compression pillars were made with focus ion beam (FIB) machining. Locally milling test structures within a single grain of a polycrystalline coupon is an established methodology and has been successfully demonstrated in $\mathrm{Al}$ by $\mathrm{Ng}$ et al. [8] and Kunz et al. [9]. The material employed for this investigation came from a large polycrystalline H-beam forging of AA2070. The material was deformation 
processed then solutionized, quenched and aged. A small coupon approximately $25 \mathrm{~mm}$ x 25 $\mathrm{mm} \times 5 \mathrm{~mm}$ was extracted from the artificially aged forging and then ground such that top and bottom faces were parallel. This coupon was then mounted to a metal puck that served as a holder for polishing as well as fixturing in a scanning electron microscope (SEM) and nanoindenter. Once mounted, the sample was polished and then orientation mapped using Quanta FEG 650 scanning electron microscope (SEM) (FEI; Hillsboro, OR) outfitted with a NordlysNano Electron Backscatter Diffraction (EBSD) detector (Oxford Instruments; Concord, MA). The orientation imaging microscopy with EBSD elucidated the crystallographic orientation and location of large grains suitable for micropillar fabrication with a FIB. The orientation mapping was performed at approximately 2,000X magnification with the electron beam set at $30 \mathrm{keV}$ and a spot size of 5 . The electron backscatter diffraction patterns were collected with 1 x 1 binning and step size of $0.5 \mu \mathrm{m}$.

A Helios Nanolab 600 dual beam microscope (FEI; Hillsboro, OR) was then employed to machine microcompression pillars into the candidate grains with a $30 \mathrm{keV}$ Ga FIB. The pillar fabrication approach followed the two step annular milling method of Volkert [10]. The target pillar geometry was a diameter of $1.5 \mu \mathrm{m}$, a height of $4 \mu \mathrm{m}$, and a taper of less than $2^{\circ}$, which follows the guidelines of Zhang et al. [11] for accurate micro-compression experiments.

After fabrication, the pillars were tested using a G200 nanoindenter (Keysight Technologies; Santa Rosa CA) outfitted with a flat diamond punch. To acquire load-deflection data for determining modulus, a series of quasi-static load-unload compression steps were performed to a total plastic strain not exceeding $10 \%$. All experiments were conducted at room temperature. Post compression SEM imaging was also performed.

\subsection{Results}

Figure 1 presents the orientation map for three different regions scanned on the AA2070. The color corresponds to the out of plane crystallographic direction and the black line segments denote boundaries for which the misorientation angle exceeds $1.5^{\circ}$. It can be seen that the AA2070 is comprised of elongated grains. Large grains with widths on the order of $20 \mu \mathrm{m}$ were selected for pillar fabrication as they are sizeable enough to accommodate the pillar footprint. Grains denoted 'A', 'B' and 'C' were selected for pillar fabrication. The pillars were milled in regions of the grain away from any boundaries. Next to each grain, a representative pillar is displayed. Two pillars in grain A were tested. This grain has the [18 14 21] orientation. Two pillars were also tested from grain B which was of the [lllll 14 orientation. A single pillar from grain $C$ was tested. This pillar was of the [ $\left.7 \begin{array}{ll}7 & 15\end{array}\right]$ orientation.

Figure 2 presents a compilation of the compressive engineering stress-strain curves for each tested pillar. The nanoindenter outputs load-displacement. To compute the strain, the approach of Frick et al. [12] was followed with the displacement adjusted by the Sneddon correction [13] to account for the elastic displacement of the base as well as any elastic deformation of the tip. For this correction, the polycrystalline AA2070 properties were taken to be elastic modulus, $\mathrm{E}=77.1 \mathrm{GPa}$ and a Poisson ratio, $v=0.31$. As for the diamond punch, $\mathrm{E}=1050 \mathrm{GPa}$ and $v=0.2$ was assumed following the property specification of the manufacturer (Micro Star Technologies; Huntsville TX).

Table 1 summaries the modulus measurements determined from the unloading curves of the compression tests. Often the first, and sometimes the second, loading step resulted in a low ( 50-60 GPa) modulus. As some pillars did not have a perfectly flat top, this artifact is assumed to stem from the indenter tip becoming fully seated on the pillar in the initial 

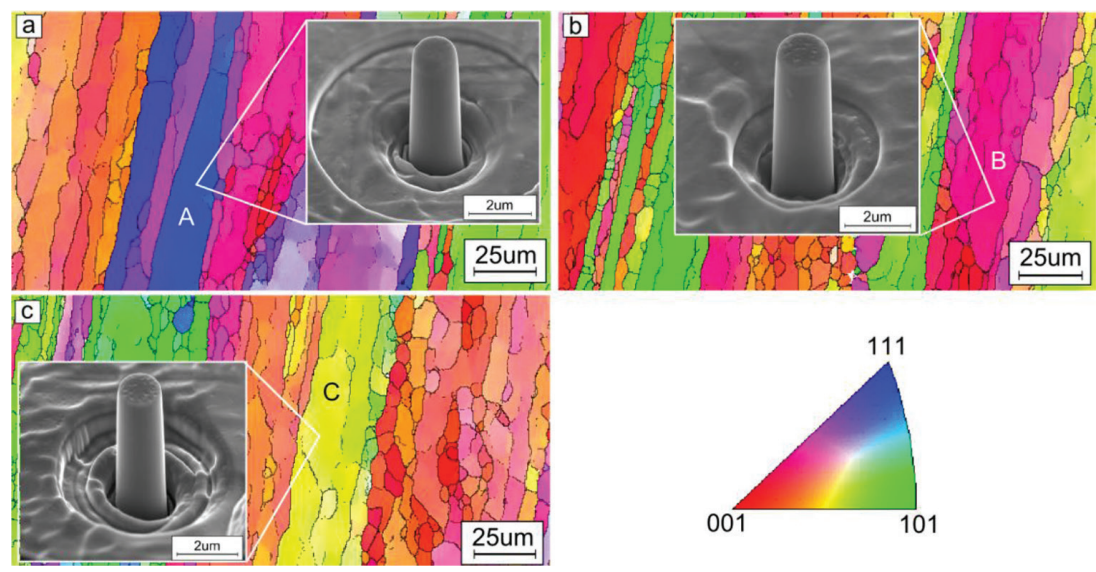

Figure 1: Out-of-plane orientation maps for three different regions (a), (b) and (c) of the AA2070 coupon. Labelled grains were those selected for pillar fabrication and the inset shows a representative pillar machined within that grain.
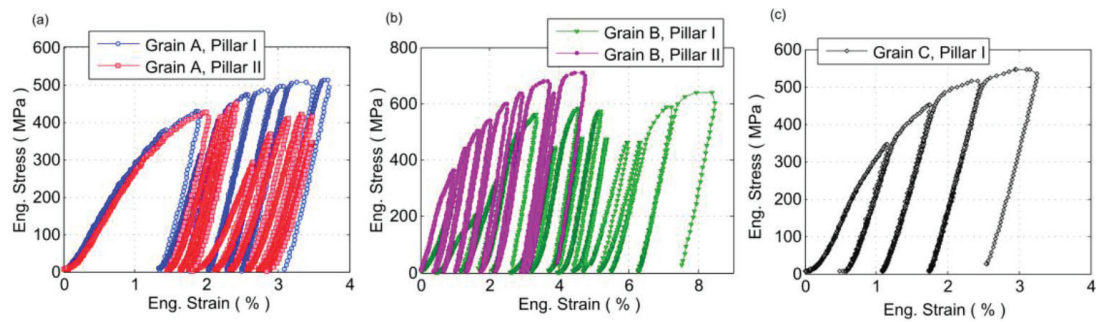

Figure 2: Compressive engineering stress-strain for (a) grian A, (b) grain B and (c) grain C.

compression cycle. These initially low modulus measurements were not considered in the reported average elastic modulus measurement. In addition, some of the modulus measurements from the latter compression cycles were discounted. For example, consider pillar II from grain A (Fig. 2a). The $5^{\text {th }}$ loading step, which has a peak stress of $300 \mathrm{MPa}$ at $2.5 \%$ strain, shows a deviation from the consistent behavior observed on the previous cycles and the modulus from this cycle was $63 \mathrm{GPa}$ which is a $22 \%$ drop compared to the previous

Table 1: Single crystal elastic modulus measurements from microcompression pillars

\begin{tabular}{llllll}
\hline Grain & Pillar ID & $\begin{array}{l}\text { Avg. Modulus } \\
(\mathrm{GPa})\end{array}$ & Std. Dev. $(\mathrm{GPa})$ & $\begin{array}{l}\text { Modulus of pure } \\
\mathrm{Al}(\mathrm{GPa})\end{array}$ & Difference \\
\hline A & I & 84.1 & 2.2 & 75.4 & $11.5 \%$ \\
A & II & 80.0 & 0.6 & & $6 \%$ \\
B & I & 78.6 & 3.4 & 67.3 & $16.8 \%$ \\
B & II & 72.0 & 3.9 & & $6.0 \%$ \\
C & I & 72.2 & 3.3 & 68.0 & $6.2 \%$ \\
\hline
\end{tabular}

*calculated from the stiffness/compliance constants of pure Al as reported in Smithells Metals Handbook. 
cycles which returned a modulus value of $80.5 \mathrm{GPa}$. It is assumed that on this loading cycle some change has occurred in loading alignment or pillar geometry. This as well as the subsequent cycles should be discounted from the average modulus.

Figure 3 highlights the state of the pillars after deformation. As the AA2070 is a face-centered-cubic system, room temperature slip will occur on the 111-type planes in the 110-directions. The 111-planes with respect to the pillar orientation are highlighted. In Fig. 3(a), a few faint slip traces are discernable and they are aligned with the slip planes orientated nearly along the axis of the pillar. For grain B (Fig. 3(b)), no slip traces were readily evident however the increase in pillar diameter and decrease in height did match the total plastic strain. Figure 3(c) highlights the deformation of the pillar in grain $\mathrm{C}$ and the traces of slip on the 111-planes are clearly observed.

\subsection{Discussion}

From the measurement of several polycrystalline aluminum alloys containing various concentrations of lithium, Noble et al. [14] reports that as a general trend, modulus increases by $\sim 6 \%$ for each wt. $\%$ of lithium present in the alloy. AA2070 contains from 1.0 to 1.4 wt.\% lithium thus compared to pure $\mathrm{Al}$, the modulus should be 6 to $8.4 \%$ higher. This polycrystalline trend appears to translate to single crystals. The measurements of this work find the modulus of AA2070 along single crystallographic directions is about $6 \%$ higher compared to pure aluminum (see Table 1). Pillar I from Grain B was found to have an exceptionally high modulus compared to the trend noted for the other pillars. It is speculated that this measurement could be influenced by the reduced sample size. Micropillar compression has been widely utilized to glean insights on the plasticity and deformation mechanisms in systems with micron and submicron length scales $[15,16]$. For this work it is the elastic behavior that is of interest; nevertheless the reduced volume of the test specimen could impact the data. AA2070 is a heat treatable system that garners strength from both solutes and precipitates. Typically, the precipitates of aluminum alloys are reported to be stiffer than the matrix metal [14] ranging from 90 to $175 \mathrm{GPa}$. If a pillar had a high volume fraction of stiff precipitates, their presence could elevate the measured modulus. Additional analysis beyond the scope of this paper is required to ascertain the exact influence of the precipitates. As a first step, follow up experiments are being planned to serial section pillars to quantify the volume fraction of precipitates. The presented tests and results aid in determining the single crystal stiffness matrix $\mathbf{C}$ (Eq. 2) and single crystal yield parameters used in the constitutive model.
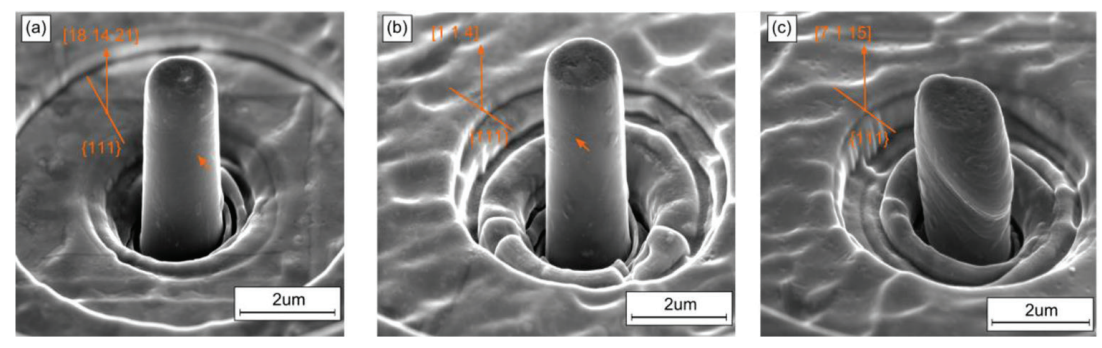

Figure 3: Representative secondary electron images of pillars after compression for (a) grain A, (b) grain B and (c) grain C. Faint slip traces are denoted with arrows and the corresponding 111-type slip plane is shown with respect to the compression axis. 


\section{TEXTURE PREDICTION UNDER FORGING CONDITIONS}

Multiscale, physics-based models can serve as valuable tools in coupling processing and manufacturing operations to local material properties. This, in turn, allows such models to support the tailoring of manufacturing processes and parameters to yield desirable structural properties as a function of microstructural features such as crystallographic texture [17]. With this goal in mind, the finite element-based crystal plasticity model presented in Section 2 was called upon to simulate the forging process of an Al-Li H-beam. The H-beam forging is approximately $1 \mathrm{~m}$ long by $0.25 \mathrm{~m}$ wide and contains regions of three different thicknesses along its length (A: $2.54 \mathrm{~cm}, \mathrm{~B}: 5.08 \mathrm{~cm}$, and C: $10.16 \mathrm{~cm}$ ). The crystal plasticity simulation utilizes calibrated parameters from the micro-, single crystal tests presented in Section 3 as well as typical values for an FCC material at the forging temperature. The slip-related model parameters used for the analyses presented in this section are the following: $\dot{\gamma}_{0}=$ $0.001 \mathrm{~s}^{-1}, \mathrm{n}=10, \mathrm{~s}_{0}=16 \mathrm{MPa}$. Based on the observed constitutive behavior of $\mathrm{Al}-\mathrm{Li}$ at the forging temperature, $\sim 450^{\circ} \mathrm{C}$, hardening was not included in the model, therefore perfect plasticity was assumed. The prescribed slip parameters govern the deformation on the 12 octahedral slip systems, $\{111\}<110>$, that are active in the FCC material. Researchers have shown that additional, non-octahedral slip systems can be active in aluminum alloys at elevated temperatures such as during hot rolling or forging [18, 19], however it has been demonstrated that deformation on the non-octahedral slip systems contributes relatively little to the final forging texture, even under triaxial forging scenarios [20].

The forging process begins with a cast ingot containing a collection of grains with no preferential orientation (i.e., randomly oriented). For the forging simulation, the initial, random microstructure is applied to a cuboid FEM mesh of 125 fully-integrated elements. Therefore the model contains 1000 integration points onto which 1000 grains with orientations sampled from a random distribution were mapped. For each integration point (i.e., grain) in the FEM mesh, the crystal plasticity model described in Section 2 is called via a usermat subroutine by ANSYS in order to solve for the deformation on each of the 12 octahedral slip systems. The aggregate of the contributions of each of the slip systems within each integration point produces the macroscale constitutive behavior observed in the material stress-strain curves. Based on the local deformation at the integration points, the corresponding grain will experience lattice rotation, thereby evolving the texture of the material as a whole.

Rolling and forging processes are typically simulated using plane strain compression boundary conditions based on the constraints applied to the material preform during its formation into the final shape. Plane strain compression of the FEM microstructure containing 1000 randomly oriented grains results in the typical Copper-type texture presented in Fig. 4. Similarly, the pole figure corresponding to a location along the mid-thickness and mid-width of the
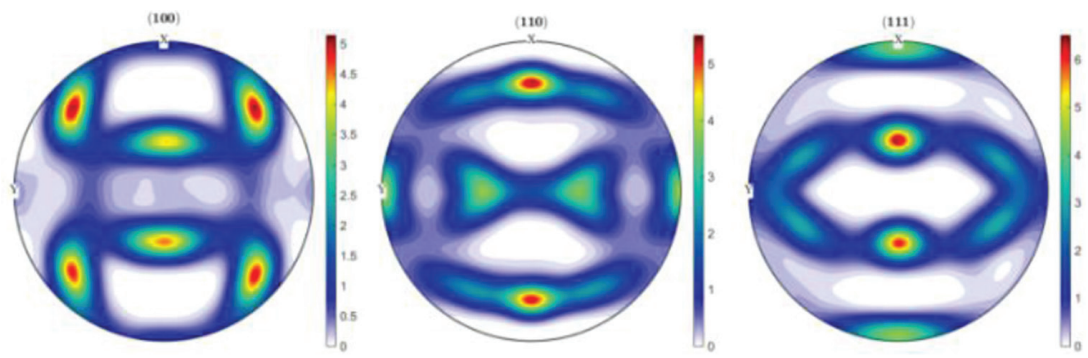

Figure 4: Copper-type texture resulting from plane strain compression of Al-Li microstructure. 
$10.16 \mathrm{~cm} \mathrm{H-beam} \mathrm{section} \mathrm{is} \mathrm{presented} \mathrm{in} \mathrm{Fig.} \mathrm{5.} \mathrm{Comparing} \mathrm{Figs} 4$ and 5, one can observe that some of the texture components are predicted correctly by the plane strain compression simulation, while others are missed entirely. Although plane strain boundary conditions are sufficient to simulate simple rolling or forging processes, it is evident from this comparison that other mechanisms are contributing to a more complicated texture profile in the case of the forged H-beam. In particular, as annotated in Fig. 5, an unusually high intensity of the Goss texture component is evident in the experimental pole figure. In addition, the pole figure in Fig. 5 appears rotated; however, this may be a result of specimen placement in the microscope.

Traditionally, the Goss texture component is associated with recrystallization. Therefore a detailed investigation was carried out on the EBSD data to determine if the presence of substantial recrystallization is responsible for the observed high-intensity Goss texture. A grain orientation spread (GOS) analysis was conducted on EBSD data taken from the $10.16 \mathrm{~cm}$ region of the H-beam. The GOS value represents an average deviation of the orientation of each point in the grain with respect to the average grain orientation. Using this approach, grains with low GOS values (e.g., $\leq 2^{\circ}$ ) can be considered recrystallized, while grains with higher values (e.g. $\geq 5^{\circ}$ ) are determined to be deformed. The results of this analysis are shown in Fig. 6. By separating the deformed grains with GOS values $\geq 5^{\circ}$ from the recrystallized grains with values $\leq 2^{\circ}$, it is possible to visualize the extent and location of recrystallization in this particular microstructure. As is evident from Fig. 6, the area fraction of recrystallized grains is small $(4.30 \%)$ compared to the area fraction of deformed grains $(70.63 \%)$. From these results, it is clear that recrystallization cannot be the primary contributor to the high-intensity Goss texture. Based on the observation that the high-intensity Goss texture can be attributed to the deformed grains, further analysis is needed to determine the specific grains responsible for this texture. Separating the grains primarily responsible for the Goss, Brass (Bs) and Copper $(\mathrm{Cu})$ texture components, we can easily observe from Fig. 7 that very long, clearly deformed grains have a Goss texture. In order to accurately predict the microstructural texture of the forged H-beam, it is therefore imperative to determine the source of the deformation-related Goss texture.

Biaxial and triaxial forging of FCC materials at elevated temperatures has been shown to alter the area fractions of various texture components [20]. For example, compression along the ST and LT directions increase the relative number of grains with the Goss orientation. It is with this knowledge that further details regarding the forging process were pursued. Although specific details of the forging operation are proprietary, we were able to deduce the general steps involved in producing the H-beam. These steps, beginning with a cast ingot,
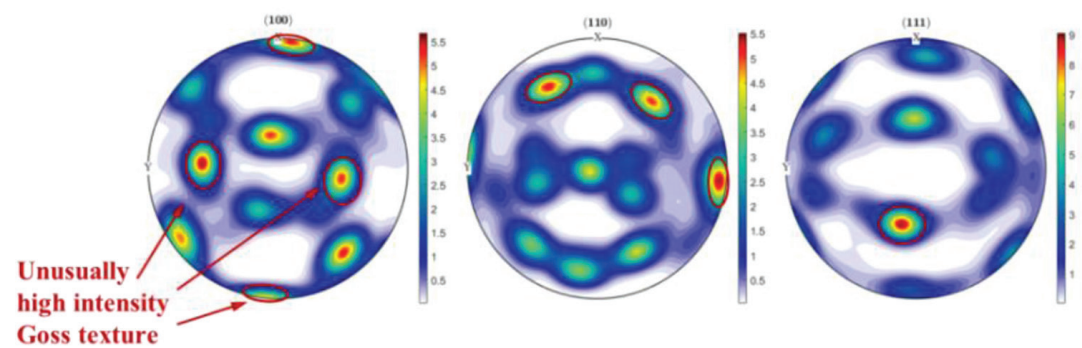

Figure 5: H-beam forging texture in Region C (10.16 cm thickness) - pole figure with regions of high Goss texture intensity highlighted. 


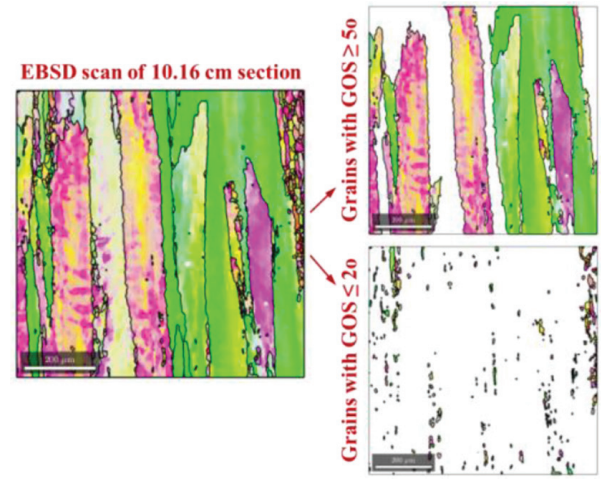

Figure 6: GOS analysis results for EBSD scan of H-beam forging.

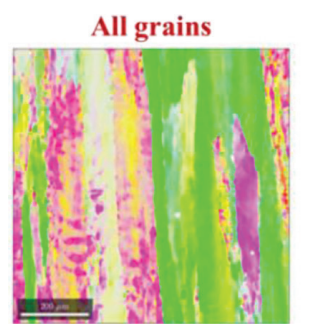

Bs-textured grains
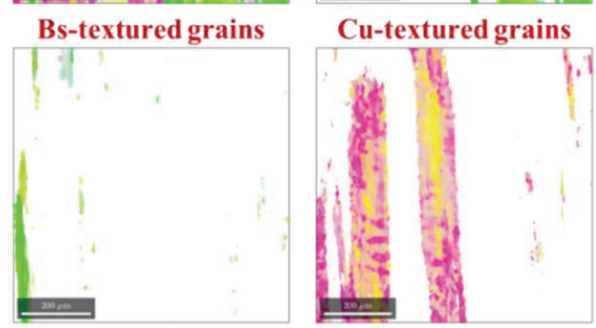

Figure 7: Grains in EBSD image separated by texture components.

include: (1) A upset, (2) B upset, (3) draw and step preform and (4) closed-die forge H-beam. These forging steps are followed by heat treatment and aging. The three steps responsible for producing the texture observed in the experimental micrographs are illustrated in Fig. 8. In order to simulate these forging steps, a three step simulation approach was utilized where the final texture from each step was applied as the initial texture of the following step, beginning with a random texture to represent the cast ingot microstructure.

Steps 1 and 2 (AB upset) were simulated as simple compression deformation, while Step 3 was approximated as plane strain compression due to the rolling/drawing-like deformation behavior. It should be noted that the A and B upsets include compression in the LT and ST directions, respectively. These two steps, in particular the A upset, intensify the Goss texture. This is in agreement with the findings of Ref. [20].

The three-step forging process was simulated using the calibrated crystal plasticity FEM model and a comparison between the simulated and forged textures is presented in Fig. 9. One can observe that all of the major texture components are represented in the pole figure of the simulated microstructure. In addition, the rotation of the pole figure is accurately predicted. It was initially assumed that the rotation observed in the experimental pole figure was a result of specimen rotation prior to EBSD imaging. However, by predicting the pole figure rotation, the simulation was able to provide otherwise-hidden insight into the deformation

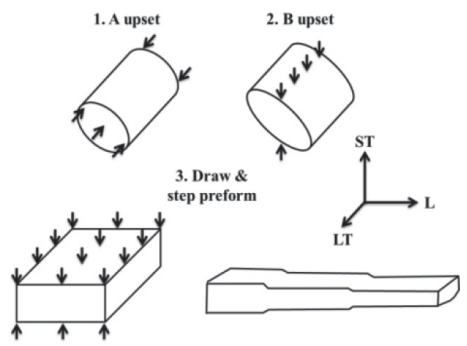

Figure 8: H-beam forging steps prior to closed-die forging into final shape. 

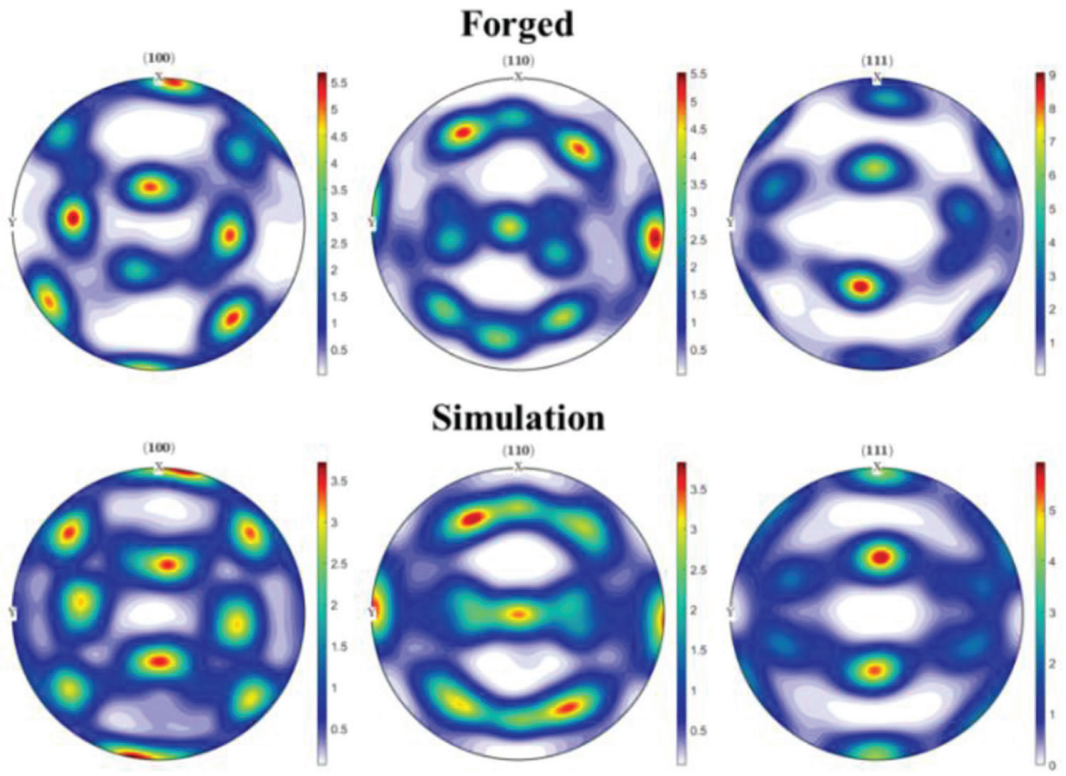

Figure 9: Pole figures of forged and simulated microstructures.
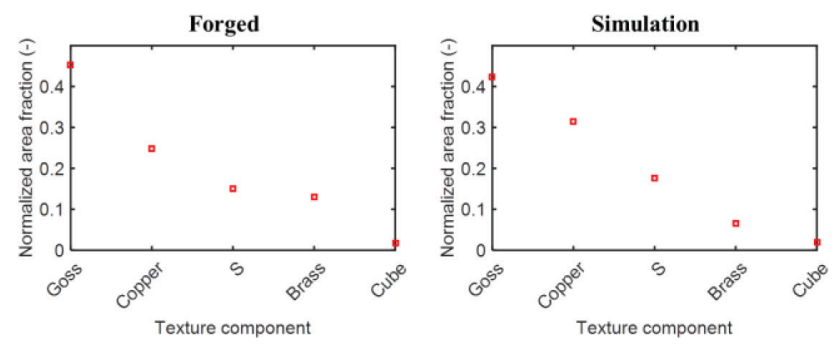

Figure 10: Texture components of forged and simulated microstructures.

behavior occurring during sequential forging operations. Analyzing the texture component area fractions, as presented in Fig. 10, allows additional quantification of the simulated forging operation. The area fractions of the Goss, Copper, S, Brass and Cube texture components are plotted due to their prevalence in rolled and forged FCC materials. The normalized area fraction of the Goss, S, and cube texture components are accurately predicted; however, the copper and brass components are over- and under-predicted, respectively. Since access to the exact strains imparted on the preform during each of the three steps is restricted due to their proprietary nature, the texture component area fractions of the simulated microstructure represent the forged microstructure accurately given the available information.

\section{CONCLUSIONS}

The goal of the developed model and forging simulation framework was to provide a tool to accurately predict the crystallographic texture of an H-beam forging. Based on experimental analysis of the EBSD data using pole figures, GOS analysis, and quantification of texture 
component volume fraction, it was determined that typical methods of simulating rolling or forging operations were insufficient to produce the observed forged texture. Considering previous effort in texture analysis of multi-axial forging operations provided guidance for accurately determining the sequential forging steps involved in producing the H-beam. These inferred steps were then verified with conversations with the forging company. In order to model the forging process, elastic properties were calibrated using micropillar tests on single crystals within the polycrystalline microstructure. These tests yielded crucial model parameters that are difficult to obtain from macroscale tests. Simulating the three-step forging process that precedes the final closed-die forging of the H-beam, we were able to accurately replicate the pole figure for material in the mid-width, mid-thickness location of the $10.16 \mathrm{~cm}$ section. Quantitative analysis of the texture component area fractions provided further validation of the developed procedure however exact replication of the area fractions was not possible since we lacked the necessary (proprietary) details of each forging step. Regardless, the developed model was demonstrated capable of accurately predicting the microstructural evolution and final texture of the H-beam, thereby satisfying a primary goal of this effort. The ability to accurately predict texture evolution during complex manufacturing processes including rolling, forging, and drawing permits the tailoring of microstructures in order to optimize directional material properties such as yield. Since one of the drawbacks of Al-Li alloys is its yield and strength anisotropy, models such as the one presented in this work can be called upon to mitigate this drawback and provide a tool to design manufacturing processes to yield more desirable material properties. This tool can therefore facilitate the design of $\mathrm{Al}-\mathrm{Li}$ structures with directional strength properties tailored to specific load scenarios similar to how composite structures are designed.

\section{ACKNOWLEDGEMENTS}

The authors are grateful for support and funding from Lightweight Innovations for Tomorrow (LIFT), operated by the American Lightweight Materials Manufacturing Innovation Institute (ALMMII).

\section{REFERENCES}

[1] Encyclopedia of Energy Engineering and Technology 1, Barney L. Capehart CRC Press. ISBN 978-0-8493-3653-9, (2007).

[2] Lee, J.J, Historical and future trends in aircraft performance, cost and emissions, MS Thesis, Massachusetts Institute of Technology, Cambridge, 2000.

[3] Gupta, R.K., Nayan, N., Nagasireesha, G. \& Sharma, S.C., Development and characterization of Al-Li alloys. Materials Science and Engineering, A420, pp. 228-234, 2006. https://doi.org/10.1016/j.msea.2006.01.045

[4] Staroselsky, A. \& Cassenti, B.N. Combined rate-independent plasticity and creep model for single crystal. Mechanics of Materials, 42(10), pp. 945-959, 2010. https://doi.org/10.1016/j.mechmat.2010.07.005

[5] Staroselsky, A., Crystal plasticity due to slip and twinning, PhD Thesis, MIT, 1997.

[6] Stouffer, D.C. \& Dame, L.T., Inelastic deformation of metals. John Wiley \& Sons, Inc, New York, 1996.

[7] Staroselsky, A. \& Cassenti, B.N., Mechanisms for tertiary creep of single crystal superalloy. Mechanics of Time-Dependent Materials, 12(4), pp. 275-289, 2008.

https://doi.org/10.1007/s11043-008-9065-6 
[8] Ng, K.S. \& Ngan, A.H.W., Stochastic nature of plasticity of aluminum micro-pillars. Acta Materialia, 56(8), pp. 1712-1720, 2008. https://doi.org/10.1016/j.actamat.2007.12.016

[9] Kunz, A., Pathak, S. \& Greer, J.R., Size effects in Al nanopillars: Single crystalline vs. bicrystalline. Acta Materialia, 59(11), pp. 4416-4424, 2011. https://doi.org/10.1016/j.actamat.2011.03.065

[10] Volkert, C.A. \& Lilleodden, E.T., Size effects in the deformation of sub-micron Au columns. Philosophical Magazine, 86(33-35), pp. 5567-5579, 2006.

https://doi.org/10.1080/14786430600567739

[11] Zhang, H., Schuster, B.E., Wei, Q. \& Ramesh, K.T., The design of accurate microcompression experiments. Scripta Materialia, 54(2), pp. 181-186, 2006. https://doi.org/10.1016/j.scriptamat.2005.06.043

[12] Frick, C.P., Clark, B.G., Orso, S., Schneider, A.S. \& Arzt, E., Size effect on strength and strain hardening of small-scale [ $\left[\begin{array}{lll}1 & 1 & 1\end{array}\right]$ nickel compression pillars. Materials Science and Engineering, A 489(1-2), pp. 319-329, 2008. https://doi.org/10.1016/j.msea.2007.12.038

[13] Sneddon, I.N., The relation between load and penetration in the axisymmetric boussinesq problem for a punch of arbitrary profile. International Journal of Engineering Science, 3(1), pp. 47-57, 1965.

https://doi.org/10.1016/0020-7225(65)90019-4

[14] Noble, B., Harris, S.J. \& Dinsdale, K., The elastic modulus of aluminium-lithium alloys. Journal of Materials Science 17(2), pp. 461-468, 1982. https://doi.org/10.1007/bf00591481

[15] Uchic, M.D., Shade, P.A. \& Dimiduk, D.M., Plasticity of micrometer-scale single crystals in compression. Annual Review of Materials Research, 39, pp. 361-386, 2009. https://doi.org/10.1146/annurev-matsci-082908-145422

[16] Greer, J.R., \& De Hosson, J.T.M., Plasticity in small-sized metallic systems: Intrinsic versus extrinsic size effect. Progress in Materials Science, 56(6), pp. 654-724, 2011. https://doi.org/10.1016/j.pmatsci.2011.01.005

[17] McDowell, D. L., A perspective on trends in multiscale plasticity. International Journal of Plasticity, 26(9), pp. 1280-1309, 2010. https://doi.org/10.1016/j.ijplas.2010.02.008

[18] Caillard, D. \& Martin, J.L., Glide of dislocations in non-octahedral planes of fcc metals: a review. International Journal of Materials Research, 100(10), pp. 1403-1410, 2009. https://doi.org/10.3139/146.110190

[19] Contrepois, Q., Maurice, C. \& Driver, J. H., Hot rolling textures of Al-Cu-Li and Al$\mathrm{Zn}-\mathrm{Mg}-\mathrm{Cu}$ aeronautical alloys: experiments and simulations to high strains. Materials Science and Engineering: A, 527(27), pp. 7305-7312, 2010. https://doi.org/10.1016/j.msea.2010.07.095

[20] Ringeval, S., Piot, D., Desrayaud, C. \& Driver, J. H., Texture and microtexture development in an $\mathrm{Al}-3 \mathrm{Mg}-\mathrm{Sc}(\mathrm{Zr})$ alloy deformed by triaxial forging. Acta Materialia, 54(11), pp. 3095-3105, 2006.

https://doi.org/10.1016/j.actamat.2006.02.047 